\title{
Stage of chronic kidney disease is an outcome-predicting factor of angioplasty for atheromatous renal artery stenosis
}

\author{
Masayuki Tanemoto, Yoichi Takeuchi, Eikan Mishima, Takehiro Suzuki, Takaaki Abe and Sadayoshi Ito \\ Angioplasty with insertion of an endoprosthesis is an effective treatment for atheromatous renal artery stenosis (ARAS). However, \\ this procedure may cause deterioration in renal function, and it is imperative to define the cases that could benefit from \\ angioplasty. From 456 suspected renovascular hypertension cases, 33 were given a diagnosis of unilateral ARAS on renal \\ arteriography. These unilateral ARAS cases were treated by angioplasty, and their baseline variables were evaluated with respect \\ to the improvement achieved in post-angioplastic renal function of the treated side as measured by renal scintigram. The \\ estimated glomerular filtration rate (eGFR) was the only variable that was significantly different between cases that showed \\ improvement in renal function and those that did not. Cases that showed improvement in renal function had lower pre- \\ angioplasty eGFR compared with cases that did not show improvement $\left(59 \pm 24 \mathrm{ml} \mathrm{min}^{-1} 1.73 \mathrm{~m}^{-2} \mathrm{vs}\right.$. $76 \pm 12 \mathrm{ml} \mathrm{min}{ }^{-1}$ \\ $\left.1.73 \mathrm{~m}^{-2}, P=0.04\right)$, and cases showing improvement were generally at later stages of chronic kidney disease (CKD). Most \\ patients without improvement, who were generally at earlier stages of CKD, had a systemic blood pressure reduction after angioplasty. \\ The present findings indicate that the baseline CKD stage could be used to predict the outcome of angioplasty for ARAS. \\ Hypertension Research (2010) 33, 1206-1210; doi:10.1038/hr.2010.152; published online 12 August 2010
}

Keywords: arteriosclerosis; chronic kidney disease; renal artery stenosis; renin angiotensin system

\section{INTRODUCTION}

Atheromatous renal artery stenosis (ARAS) accounts for $70-90 \%$ of stenotic lesions of the renal artery. ${ }^{1,2}$ By impairing renal perfusion, ARAS primarily causes two clinical symptoms: renal impairment by ischemic renal disease and hypertension by renovascular hypertension. However, renal impairment and hypertension could facilitate atheromatous vascular disease, and incidental finding of ARAS without perfusion impairment is not rare in hypertensive patients. ${ }^{3,4}$ Although percutaneous transluminal renal angioplasty with insertion of stainless steel endoprosthesis (PTRA-S) could improve renal function and reduce the systemic blood pressure (BP) in ARAS cases with perfusion impairment, ${ }^{2,5}$ the procedure during PTRA-S may deteriorate renal function. ${ }^{6,7}$ Therefore, it is imperative to define the cases that could benefit from PTRA-S treatment.

Previously, we reported that the hemodynamic variables in ARAS could indicate cases that would have BP reduction by PTRA-S treatment. ${ }^{8}$ We showed that the cases could be selected on the basis of the value of the trans-stenotic pressure gradient (PG) at ARAS divided by its corresponding pre-stenotic pressure (PG/pre). However, we were unable to define the cases that would have renal function improvement after PTRA-S treatment because of the difficulty in evaluating the improvement. Parameters such as serum creatinine concentration and estimated glomerular filtration rate (eGFR) could not indicate the improvement accurately, because their values would fluctuate solely because of repeated measurement. These parameters also might be decreased by several factors during PTRA-S treatment, including contrast nephropathy and atheroembolization, ${ }^{9}$ even if PTRA-S treatment improved the function of the ARAS-affected region.

In this study, we used 99mTc-diethylenetriamine-pentaacetic acid (99mTc-DTPA) renal scintigrams to measure glomerular filtration rate (DTPA-GFR) on each side of the kidney in patients with unilateral ARAS. We evaluated baseline variables of the patients with reference to post-angioplastic increase in the relative DTPA-GFR of the side affected by ARAS (ARAS side), which was calculated as the DTPA-GFR of the ARAS side divided by the DTPA-GFR of the contralateral side.

\section{METHODS}

Subjects

Among the hypertensive cases referred to our department between April 2003 and June 2008, 456 cases with at least one atheromatous risk factor (smoking, dyslipidemia or DM) and preserved renal function (serum creatinine concentration $<2.0 \mathrm{mg} \mathrm{dl}^{-1}$ ) were evaluated by computed tomographic angiography 
or magnetic resonance angiography. The cases with ARAS on computed tomographic angiography/magnetic resonance angiography were further evaluated by selective renal arteriography. The severity of ARAS was indicated by the width at the narrowest part and the \% diameter reduction, which was calculated as $100 \times\left(1\right.$-narrowest part width/reference width).${ }^{10}$ In all, 33 cases with unilateral ARAS of the narrowest part width $<3 \mathrm{~mm}$ or a $\%$ diameter reduction $>50 \%$ were included in this study. In all cases, ARAS was treated using PTRA-S (Palmaz stent, diameter: 5-7 mm). The study protocol was approved by the ethics committee of our hospital, and informed consent was obtained from all patients.

\section{Evaluation of renal function}

The $99 \mathrm{mTc}$-DTPA renal scintigrams were performed by a commercially available $\gamma$-camera system (PRISM-IRIX, Shimadzu, Kyoto, Japan), and DTPA-GFR was measured from each side of the kidney according to the manufacturer's instructions. The relative DTPA-GFR was calculated as the DTPA-GFR of the ARAS side divided by that of the contralateral side and used as a parameter for the renal function of the ARAS side. For each case, the renal scintigram was performed before and 1 week after PTRA-S treatment, and the relative DTPA-GFR after PTRA-S treatment was compared with that before the procedure.

According to the values of the relative DTPA-GFR, the cases were divided into two groups: the GFR-increased group and the GFR-nonincreased group. The cases that had the relative DTPA-GFR after PTRA-S treatment larger than that before the procedure were included in the GFR-increased group. All other cases were included in the GFR-nonincreased group.

\section{Baseline and follow-up studies}

The baseline and post-angioplastic information was recorded under the effect of antihypertensive medication. For PTRA-S treatment, the baseline information was recorded at the time of admission, and the post-angioplastic information was recorded approximately 1 month after the procedure. All blood samples were collected in morning under fasting conditions. Albuminuria was measured as the spot urinary albumin to creatinine ratio, and eGFR was calculated by the GFR-estimating equations for Japanese individuals. ${ }^{11}$ The office BP reading was taken according to the standard guidelines. ${ }^{12}$ The BP self-monitoring was also performed ${ }^{13,14}$ and correlated well with the office BP.

$\mathrm{BP}$ reduction was defined as post-angioplastic reduction in the total score of the administered antihypertensive agents, which was calculated as the sum of the standardized doses (the administered dose divided by its corresponding maximum approved daily dose). ${ }^{8}$ For the case with the total score reduction, it was confirmed that neither the office BP nor the self-monitored BP increased after PTRA-S treatment.

\section{Pressure measurement at ARAS lesion sites}

Arterial pressure was measured at each ARAS lesion site with an end-hole 3-French catheter. ${ }^{8,15}$ The pressure levels measured distal and proximal to the lesion were recorded as the post-stenotic and the pre-stenotic pressure, respectively. PG was calculated as the post-stenotic pressure subtracted from its corresponding pre-stenotic pressure. PG/pre was calculated as PG divided by its corresponding pre-stenotic pressure.

\section{Renal Doppler ultrasonography}

Renal Doppler ultrasonography was performed with a commercially available ultrasound machine (AplioXV, Toshiba, Tochigi, Japan). Images of the kidney were acquired before PTRA-S treatment in a supine or lateral position with a 3.5-MHz pulsed Doppler frequency transducer, and the pole-to-pole length on the images was measured as the size of the kidney. Intrarenal Doppler signals were obtained from interlobar arteries. The peak systolic velocity and the enddiastolic velocity were measured at each artery, and resistive index (RI) was calculated as (1-end-diastolic velocity)/peak systolic velocity.

\section{Statistical analysis}

Continuous variables are expressed as mean \pm s.d. Between-group comparisons were analyzed by the Student's $t$-test. Discrete variables were expressed as counts, and comparisons were analyzed using the $\chi^{2}$-test. All statistical analyses were performed on the Dr SPSS II software (SPSS, Chicago, IL, USA), and probability values of $P<0.05$ were considered statistically significant.

\section{RESULTS}

The baseline characteristics of the cases examined in this study are summarized in Table 1. PTRA-S treatment increased the relative DTPA-GFR in 22 cases (the DTPA-GFR-increased group) but not in the other 11 cases (the DTPA-GFR-nonincreased group). The DTPA-GFR-increased group had lower eGFR than the DTPA-GFRnonincreased group, and eGFR was the only baseline characteristic that was significantly different between the groups. The difference was significant regardless of whether eGFR was calculated by the Cockroft and Gault formula (C-G formula); however, the eGFR of the $\mathrm{C}-\mathrm{G}$ formula was generally higher than the values obtained from the formula used in the Modification of Diet in Renal Disease study. The eGFRs of the C-G formula were $71 \pm 28 \mathrm{ml} \mathrm{min}^{-1}$ and $97 \pm 19 \mathrm{ml} \mathrm{min}^{-1}(P=0.01)$ in the DTPA-GFR-increased group and the DTPA-GFR-nonincreased group, respectively. The other baseline characteristics examined were not significantly different between the groups. Both groups were given approximately the same amount and kind of antihypertensive medication. In the cases examined by renal ultrasonography, the size of the kidney was also compared between the groups. We found that the size of the ARAS side and that of the contralateral side were not significantly different between the groups (Table 2).

Further analysis of the baseline eGFR revealed that the DTPA-GFRincreased group was generally at later stages of chronic kidney disease (CKD) compared with the DTPA-GFR-nonincreased group (Figure 1). ${ }^{16,17}$ Fourteen (14 out of 22) and 7 (7 out of 22) cases of the DTPA-GFR-increased group were at stage 3 (eGFR 30$\left.59 \mathrm{ml} \mathrm{min}^{-1} 1.73 \mathrm{~m}^{-2}\right)$ and at stages $1-2\left(\right.$ eGFR $\geqslant 60 \mathrm{ml} \mathrm{min}^{-1}$ $1.73 \mathrm{~m}^{-2}$ ), respectively. In contrast, 1 ( 1 out of 11$)$ and 10 (10 out of 11) cases of the DTPA-GFR-nonincreased group were at stage 3 and at stages $1-2$, respectively. In all, 14 of 15 cases at stage 3 and 7 of 17 cases at stages 1-2 had an increase in DTPA-GFR.

The severity in the impairment of renal perfusion at ARAS and its improvement by PTRA-S treatment could have affected the DTPAGFR increase. Therefore, we next examined the hemodynamic variables of ARAS (Table 3). None of the examined variables were significantly different between the groups, and PTRA-S treatment decreased the systolic PG to $<10 \mathrm{~mm} \mathrm{Hg}$ in all the cases, indicating that PTRA-S treatment improved the perfusion at the ARAS lesion site in the DTPA-GFR-nonincreased group as well as in the DTPA-GFRincreased group. Furthermore, among the cases examined by renal Doppler ultrasonography, the RIs of the interlobar arteries in both the ARAS side and the contralateral side were also not significantly different between the groups (Table 2).

The post-angioplastic characteristics examined 1 month after PTRA-S treatment are summarized in Table 4. After PTRA-S treatment, eGFR decreased not only in the DTPA-GFR-nonincreased group but also in the DTPA-GFR-increased group. The eGFR values did not increase in 26 cases of this study. Moreover, the postangioplastic eGFR in the DTPA-GFR-increased group remained significantly lower than that in the nonincreased group. The reduction in eGFR and the relative regression from the baseline eGFR were the same in both groups ( $3 \pm 14 \%$, respectively).

Interestingly, the PRA in the DTPA-GFR-increased group, which had been generally higher before PTRA-S treatment, was generally lower after the procedure compared with the DTPA-GFR-nonincreased group. This change in PRA would indicate that PTRA-S treatment generally reduced PRA more efficiently in the former 
Table 1 Baseline characteristics of the cases

\begin{tabular}{|c|c|c|c|c|}
\hline & \multirow[b]{2}{*}{ All $(\mathrm{n}=33)$} & \multicolumn{2}{|c|}{ DTPA-GFR } & \multirow[b]{2}{*}{$\mathrm{P}$} \\
\hline & & $\begin{array}{c}\text { Increased } \\
(\mathrm{n}=22)\end{array}$ & $\begin{array}{c}\text { Nonincreased } \\
(\mathrm{n}=11)\end{array}$ & \\
\hline Age (years) & $57 \pm 8$ & $58 \pm 8$ & $56 \pm 8$ & 0.41 \\
\hline Gender (\% female) & 32 & 29 & 39 & 0.19 \\
\hline Body mass index $\left(\mathrm{kg} \mathrm{m}^{-2}\right)$ & $24 \pm 3$ & $24 \pm 3$ & $24 \pm 3$ & 0.93 \\
\hline \multicolumn{5}{|l|}{ Smoking status, $n(\%)$} \\
\hline Never & $7(21)$ & $5(23)$ & $2(18)$ & \\
\hline Past & $11(33)$ & $7(32)$ & $4(36)$ & \\
\hline Current & $15(45)$ & $10(45)$ & $5(45)$ & 1.00 \\
\hline \multicolumn{5}{|l|}{ Systemic blood pressure $(\mathrm{mm} \mathrm{Hg})$} \\
\hline Systolic & $130 \pm 18$ & $130 \pm 18$ & $131 \pm 19$ & 0.87 \\
\hline Diastolic & $75 \pm 10$ & $74 \pm 11$ & $78 \pm 9$ & 0.28 \\
\hline \multicolumn{5}{|l|}{ Antihypertensive, $n$ (\%) } \\
\hline Calcium channel blocker & $31(94)$ & $21(95)$ & $10(91)$ & 0.61 \\
\hline ACEI/ARB & $24(73)$ & $16(73)$ & $8(73)$ & 1.00 \\
\hline$\beta$-Blocker & $5(15)$ & $3(14)$ & $2(18)$ & 0.73 \\
\hline$\alpha$-Blocker & $6(18)$ & $4(18)$ & $2(18)$ & 1.00 \\
\hline Diuretic & $7(21)$ & $5(23)$ & $2(18)$ & 0.76 \\
\hline \multicolumn{5}{|l|}{ Lipid profile (mgdl-1) } \\
\hline Triglyceride & $120 \pm 52$ & $121 \pm 60$ & $116 \pm 34$ & 0.79 \\
\hline HDL cholesterol & $46 \pm 10$ & $45 \pm 8$ & $48 \pm 12$ & 0.41 \\
\hline LDL cholesterol & $119 \pm 33$ & $117 \pm 36$ & $122 \pm 26$ & 0.64 \\
\hline $\mathrm{HbA1c}(\%)$ & $5.4 \pm 0.5$ & $5.4 \pm 0.6$ & $5.3 \pm 0.3$ & 0.42 \\
\hline eGFR $\left(\mathrm{ml} \mathrm{min}-11.73 \mathrm{~m}^{-2}\right)$ & $65 \pm 22$ & $59 \pm 24$ & $76 \pm 12$ & 0.04 \\
\hline Plasma renin activity $\left(\mathrm{ng} \mathrm{ml}^{-1} \mathrm{~h}^{-1}\right)$ & $7.1 \pm 6.3$ & $7.7 \pm 6.9$ & $6.0 \pm 5.0$ & 0.50 \\
\hline Albumin-creatinine ratio $\left(\mathrm{mgg}^{-1}\right)$ & $45 \pm 67$ & $49 \pm 79$ & $35 \pm 32$ & 0.58 \\
\hline
\end{tabular}

Table 2 Renal Doppler ultrasonography

\begin{tabular}{lcccc}
\hline & & \multicolumn{2}{c}{ DTPA-GFR } & \\
\cline { 3 - 4 } & All $(\mathrm{n}=19)$ & $\begin{array}{c}\text { Increased } \\
(\mathrm{n}=11)\end{array}$ & $\begin{array}{c}\text { Nonincreased } \\
(\mathrm{n}=8)\end{array}$ & $\mathrm{P}$ \\
\hline Size $(\mathrm{mm})$ & & & & \\
$\quad$ ARAS side & $93 \pm 14$ & $89 \pm 15$ & $100 \pm 11$ & 0.11 \\
Contralateral & $104 \pm 14$ & $101 \pm 15$ & $109 \pm 11$ & 0.23 \\
RI & & & & \\
ARAS side & $0.58 \pm 0.09$ & $0.60 \pm 0.09$ & $0.57 \pm 0.08$ & 0.48 \\
Contralateral & $0.61 \pm 0.06$ & $0.62 \pm 0.08$ & $0.59 \pm 0.04$ & 0.33 \\
\hline
\end{tabular}

Abbreviations: ARAS, atheromatous renal artery stenosis; DTPA-GFR, 99mTc-diethylenetriaminepentaacetic acid-glomerular filtration rate measured by renal scintigram; RI, resistive index of interlobar arteries.

Data are presented as mean \pm s.d.

than in the latter group. In contrast to the PRA change, a reduction in BP, which was indicated by a reduction in the total score of the administered antihypertensive agents, was noted more in the

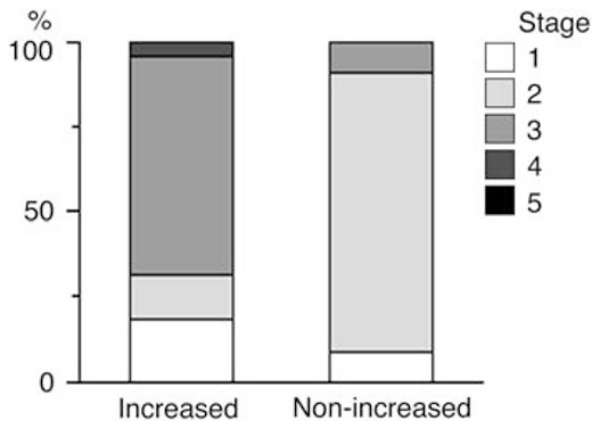

Figure 1 The chronic kidney disease stage in the 99mTc-diethylenetriaminepentaacetic acid-glomerular filtration rate-increased group (left) and the $99 \mathrm{mTc}$-diethylenetriamine-pentaacetic acid-glomerular filtration ratenonincreased group (right).

Table 3 Hemodynamic variables of ARAS lesion sites

\begin{tabular}{lcccc}
\hline & & \multicolumn{2}{c}{ DTPA-GFR } \\
\cline { 3 - 4 } & & $\begin{array}{c}\text { Increased } \\
(\mathrm{n}=22)\end{array}$ & $\begin{array}{c}\text { Nonincreased } \\
(\mathrm{n}=11)\end{array}$ & \\
\hline$P G(\mathrm{~mm} \mathrm{Hg})$ & & & & \\
$\quad$ Systolic & $65 \pm 38$ & $68 \pm 41$ & $59 \pm 32$ & 0.51 \\
Mean & $37 \pm 25$ & $40 \pm 27$ & $33 \pm 22$ & 0.50 \\
Diastolic & $21 \pm 17$ & $21 \pm 17$ & $20 \pm 19$ & 0.81 \\
& & & & \\
$P G /$ pre & & & & 0.69 \\
Systolic & $0.42 \pm 0.24$ & $0.43 \pm 0.25$ & $0.40 \pm 0.21$ & 0.65 \\
Mean & $0.35 \pm 0.23$ & $0.36 \pm 0.25$ & $0.32 \pm 0.20$ & 0.75 \\
Diastolic & $0.27 \pm 0.24$ & $0.28 \pm 0.24$ & $0.25 \pm 0.24$ & 0.77 \\
\hline
\end{tabular}

Abbreviations: ARAS, atheromatous renal artery stenosis; DTPA-GFR, 99mTc-

diethylenetriamine-pentaacetic acid-glomerular filtration rate measured by renal scintigram; PG, trans-stenotic pressure gradient; pre, pre-stenotic pressure.

Data are presented as mean \pm s.d.

Table 4 Post-angioplastic characteristics of the cases

\begin{tabular}{|c|c|c|c|c|}
\hline & \multirow[b]{2}{*}{$A / /(\mathrm{n}=33)$} & \multicolumn{2}{|c|}{ DTPA-GFR } & \multirow[b]{2}{*}{$\mathrm{P}$} \\
\hline & & $\begin{array}{l}\text { Increased } \\
(\mathrm{n}=22)\end{array}$ & $\begin{array}{l}\text { Nonincreased } \\
\quad(\mathrm{n}=11)\end{array}$ & \\
\hline eGFR (ml min $\left.\min ^{-1} 1.73 \mathrm{~m}^{-2}\right)$ & $62 \pm 21$ & $56 \pm 22$ & $72 \pm 12$ & 0.03 \\
\hline Reduction from baseline & $3 \pm 10$ & $2 \pm 9$ & $3 \pm 12$ & 0.84 \\
\hline Plasma renin activity $\left(\mathrm{ng} \mathrm{ml}^{-1} \mathrm{~h}^{-1}\right)$ & $5.3 \pm 9.0$ & $5.2 \pm 10.1$ & $5.5 \pm 6.5$ & 0.94 \\
\hline Reduction from baseline & $1.8 \pm 9.4$ & $2.4 \pm 10.7$ & $0.6 \pm 6.3$ & 0.60 \\
\hline \multicolumn{5}{|c|}{$\begin{array}{l}\text { Abbreviations: DTPA-GFR, 99mTc-diethylenetriamine-pentaacetic acid-glomerular filtration rate } \\
\text { measured by renal scintigram; eGFR, estimated glomerular filtration rate calculated by the } \\
\text { Modification of Diet in Renal Disease study formula. } \\
\text { Data are presented as mean } \pm \text { s.d. }\end{array}$} \\
\hline
\end{tabular}

DTPA-GFR-nonincreased group than in the DTPA-GFR-increased group ( 10 out of 11 cases in the former and 12 out of 22 cases in the latter had the reduction).

\section{DISCUSSION}

In this study, we evaluated the increase in DTPA-GFR after PTRA-S treatment to indicate the effect of the procedure, because generally used indicators of renal function, such as serum creatinine concentration and 
eGFR, were unable to accurately assess the effect. ${ }^{9}$ Using this indication, we evaluated the baseline characteristics of ARAS cases, including age, metabolic profile, smoking status, BP control and albuminuria, and found that the baseline eGFR value was the only characteristic that was significantly correlated with the effect. We also found that many cases at CKD stage 3 had an increase in DTPA-GFR after PTRA$S$ treatment. Moreover, many cases at CKD stages $1-2$ had a reduction in BP after PTRA-S treatment. These findings indicate that the baseline CKD stage, which represents the baseline eGFR value, could be used to predict the outcome of PTRA-S treatment for ARAS.

The correlation between the baseline eGFR and the angioplastic outcome has previously been reported. ${ }^{18}$ At 1 year after PTRA-S treatment, eGFR (calculated by the $\mathrm{C}-\mathrm{G}$ formula) was found to be increased in cases with a baseline eGFR $<50 \mathrm{ml} \mathrm{min}^{-1}$, whereas it decreased in those with a baseline eGFR $\geqslant 50 \mathrm{mlmin}^{-1}$. These findings indicated that cases with decreased baseline eGFR could have long-term renal preservation after PTRA-S treatment. Most cases in this study had a baseline eGFR $\geqslant 50 \mathrm{ml} \mathrm{min}^{-1}$ (using the $\mathrm{C}-\mathrm{G}$ formula) and were consistent with the eGFR-decreased subgroup of the previous report. Therefore, the finding that most cases in this study had a decrease in eGFR 1 month after PTRA-S treatment, which is consistent with a reduction in renal function 1 year after PTRA-S treatment, may support the previous findings.

Interestingly, despite the increase in DTPA-GFR after PTRA-S treatment, eGFR decreased 1 month after the procedure in both the DTPA-GFR-increased and nonincreased groups. This decrease of eGFR indicates that some factors during PTRA-S treatment, for example, contrast nephropathy and atheroembolization, deteriorated long-term renal function, ${ }^{9}$ even though PTRA-S treatment could have improved the renal function immediately after angioplasty. These renal function-deteriorating factors may have also concealed the benefit of angioplasty in the previous reports, in which angioplasty did not show significant clinical benefits in ARAS cases. ${ }^{19,20}$ Protection against these factors during the procedure might improve the longterm reno-preservative effect by angioplasty, especially in cases with increased DTPA-GFR after angioplasty.

In this study, the eGFR calculated using the Modification of Diet in Renal Disease study formula was generally lower than that obtained using the $\mathrm{C}-\mathrm{G}$ formula. Therefore, the reported cases with an eGFR $<50 \mathrm{ml} \mathrm{min}^{-1}$ calculated using the $\mathrm{C}-\mathrm{G}$ formula might have an eGFR $<30 \mathrm{ml} \mathrm{min}^{-1} 1.73 \mathrm{~m}^{-2}$ using the Modification of Diet in Renal Disease study formula, and the finding in the report by Ramos et al. ${ }^{18}$ could have indicated that cases at CKD stages $4-5$ had an increase in eGFR 1 year after PTRA-S treatment. We could not confirm this finding because we did not include most of the cases at CKD stages $4-5$ to avoid possible adverse events such as contrast nephropathy and nephrogenic systemic fibrosis. ${ }^{21}$

The increase in DTPA-GFR represents recovery of perfusion to renal parenchyma. Supporting this notion, reduction of PRA, which would reflect recovery of the parenchymal perfusion, ${ }^{22}$ was generally larger in the DTPA-GFR-increased group than in the DTPA-GFRnonincreased group. Given that PTRA-S equally reduced PG at ARAS lesion sites in both groups, perfusion recovery at these sites would not have affected the increase in DTPA-GFR. ${ }^{7,8,15,23}$ In addition, because the RIs (an index for vascular resistance) of interlobar arteries were not different between the groups, ${ }^{1,2,24}$ perfusion impairment in these arteries also would not have affected the increase in DTPA-GFR. The perfusion impairment in more distal arteries may have affected the increase.

A high intrarenal RI $(>0.80)$ was reported to identify ARAS cases in which angioplasty will not improve renal function. ${ }^{24}$ However, this finding has not been confirmed in other studies, which instead have found no difference in renal function outcome between cases with intrarenal RI $>0.80$ and those with intrarenal RI $<0.80 .^{25,26}$ One of these studies showed that cases with deteriorated baseline renal function tended to have renal function improvement after PTRA-S treatment independent of intrarenal RI. ${ }^{25}$ The present finding that cases at CKD stages 1-3 did not have long-term reno-preservation despite the low intrarenal RI $(<0.80)$ supports the findings of Zeller et al. ${ }^{25}$

The parenchymal perfusion would have been preserved before PTRA-S treatment in the DTPA-GFR-nonincreased group, in which most cases were at CKD stages $1-2 . \cdot^{16,17}$ Given that ARAS had impaired the perfusion before PTRA-S treatment in both groups, ${ }^{7,8,15,23}$ some supplementary pathways for blood supply such as the collateral arteries would have preserved the perfusion in the group. In contrast, in the DTPA-GFR-increased group, supplementary blood supply might not have been sufficient for the preservation, and PTRA-S treatment could have improved the perfusion. In general, the late stages of CKD in the DTPA-GFR-increased group might have represented this insufficiency. The size of the ARAS side, which was generally smaller in the DTPA-GFR-increased group than in the DTPA-GFR-nonincreased group, might also have represented this insufficiency. Given that PTRA-S treatment reduced the BP efficiently in the DTPA-GFR-nonincreased group, further recovery of the perfusion preserved by supplementary blood supply could have contributed to the BP reduction but not to the increase in DTPA-GFR.

This study has several limitations. First, our study was conducted in a small number of cases screened by criteria such as preserved renal function. The paucity and the selection bias only allowed for limited interpretations. Next, the follow-up duration was only 1 month after PTRA-S treatment, and long-term reno-preservation by the procedure was not addressed. However, long-term reno-preservation would not be expected without immediate reno-preservation, which was indicated by the relative increase in DTPA-GFR. Finally, the usage of the relative DTPA-GFR could have affected the interpretation of renal function improvement. However, we used the relative DTPA-GFR value because it could indicate renal function improvement by angioplasty better than the absolute values of DTPA-GFR and eGFR. The absolute values of DTPA-GFR and eGFR did not accurately represent the improvement because they were solely affected by the conditions of evaluation (for example, dehydration could decrease them and overhydration could cause an increase). These values may also be affected by several other factors during PTRA-S treatment, including contrast nephropathy. ${ }^{9}$ In contrast, showing the renal function of the treated side relative to that of the untreated side, the relative DTPA-GFR can represent the improvement, as it could cancel out the effects of the factors that might conceal the improvement by affecting both sides.

In conclusion, we found that CKD stage could predict renopreservation by PTRA-S treatment for ARAS. We previously found that an angiographic index, the minimal diameter, could represent the hemodynamic significance of ARAS and that a hemodynamic index, PG/pre, could predict the BP reduction by PTRA-S treatment for ARAS. ${ }^{8,10}$ Therefore, in combination with previous findings, the findings of this study present the strategy for ARAS described below. For reno-preservation, PTRA-S should be performed in cases at CKD stages 4-5 regardless of the hemodynamic significance. PTRA$S$ will not likely preserve renal function in cases at CKD stages 1-3; however, it may be beneficial if performed with appropriate protection against renal function-deteriorating factors, especially in cases at CKD stage 3. Finally, for BP reduction (especially in cases at CKD stages 
1-2), the minimal diameter of ARAS should be measured. In cases with a minimal diameter $<3 \mathrm{~mm}$, PG should be measured and PTRA$S$ treatment should be given if $\mathrm{PG} /$ pre $>0.15$. Although the paucity and the selection bias of this study did not yield convincing evidence, the present findings propose a useful strategy using the CKD stage for future trials in this field.

\section{CONFLICT OF INTEREST}

The authors declare no conflict of interest.

\section{ACKNOWLEDGEMENTS}

This work was supported by Grants-in-Aid for Scientific Research from the Ministry of Education, Culture, Sports, Science and Technology of Japan (MT and TA). The study was also supported by grants from the Salt Science Research Foundation (No. 09C2) (MT) and the Sankyo Foundation of Life Science (MT).

1 Safian RD, Textor SC. Renal-artery stenosis. N Engl J Med 2001; 344: 431-442.

2 Bokhari SW, Faxon DP. Current advances in the diagnosis and treatment of renal artery stenosis. Rev Cardiovasc Med 2004; 5: 204-215.

3 Tanemoto M, Saitoh H, Satoh F, Satoh H, Abe T, Ito S. Predictors of undiagnosed renal artery stenosis among Japanese patients with risk factors of atherosclerosis. Hypertens Res 2005; 28: 237-242.

4 Uzu T, Takeji M, Yamada N, Fujii T, Yamauchi A, Takishita S, Kimura G. Prevalence and outcome of renal artery stenosis in atherosclerotic patients with renal dysfunction. Hypertens Res 2002; 25: 537-542.

5 Blum U, Krumme B, Flugel P, Gabelmann A, Lehnert T, Buitrago-Tellez C, Schollmeyer $\mathrm{P}$, Langer M. Treatment of ostial renal-artery stenoses with vascular endoprostheses after unsuccessful balloon angioplasty. N Engl J Med 1997; 336: 459-465.

6 Textor SC, Wilcox CS. Renal artery stenosis: a common, treatable cause of renal failure?. Annu Rev Med 2001; 52: 421-442.

7 Gross CM, Kramer J, Weingartner O, Uhlich F, Luft FC, Waigand J, Dietz R. Determination of renal arterial stenosis severity: comparison of pressure gradient and vessel diameter. Radiology 2001; 220: 751-756.

8 Tanemoto M, Suzuki T, Abe M, Abe T, Ito S. Hemodynamic index of atheromatous renal artery stenosis for angioplasty. Clin J Am Soc Nephrol 2009; 4: 651-655.

9 Tepel M, Aspelin P, Lameire N. Contrast-induced nephropathy: a clinical and evidence-based approach. Circulation 2006; 113: 1799-1806.

10 Tanemoto M, Abe M, Uruno A, Abe T, Ito S. Angiographic index for angioplasty-treatable atheromatous renal artery stenosis. Hypertens Res 2008; 31: 881-885.
11 Matsuo S, Imai E, Horio M, Yasuda Y, Tomita K, Nitta K, Yamagata K, Tomino Y, Yokoyama $\mathrm{H}$, Hishida A. Revised equations for estimated GFR from serum creatinine in Japan. Am J Kidney Dis 2009; 53: 982-992.

12 Rundback JH, Sacks D, Kent KC, Cooper C, Jones D, Murphy T, Rosenfield K, White C, Bettmann M, Cortell S, Puschett J, Clair D, Cole P. Guidelines for the reporting of renal artery revascularization in clinical trials American Heart Association. Circulation 2002; 106: 1572-1585.

13 Stergiou GS, Efstathiou SP, Alamara CV, Mastorantonakis SE, Roussias LG. Home or self blood pressure measurement?. What is the correct term? J Hypertens 2003; 21: 2259-2264.

14 Myers MG, Tobe SW, McKay DW, Bolli P, Hemmelgarn BR, McAlister FA. New algorithm for the diagnosis of hypertension. Am J Hypertens 2005; 18: 1369-1374.

15 De Bruyne B, Manoharan G, Pijls NH, Verhamme K, Madaric J, Bartunek J, Vanderheyden M, Heyndrickx GR. Assessment of renal artery stenosis severity by pressure gradient measurements. J Am Coll Cardiol 2006; 48: 1851-1855.

$16 \mathrm{~K} / \mathrm{DOQ}$ clinical practice guidelines for chronic kidney disease: evaluation classification stratification. Am J Kidney Dis 2002; 39: S1-S266.

17 Levey AS, Eckardt KU, Tsukamoto Y, Levin A, Coresh J, Rossert J, De Zeeuw D, Hostetter TH, Lameire N, Eknoyan G. Definition and classification of chronic kidney disease: a position statement from kidney disease: improving global outcomes (KDIGO). Kidney Int 2005; 67: 2089-2100.

18 Ramos F, Kotliar C, Alvarez D, Baglivo H, Rafaelle P, Londero H, Sanchez R, Wilcox CS. Renal function and outcome of PTRA and stenting for atherosclerotic renal artery stenosis. Kidney Int 2003; 63: 276-282.

19 van Jaarsveld BC, Krijnen P, Pieterman H, Derkx FH, Deinum J, Postma CT, Dees A, Woittiez AJ, Bartelink AK, Man in 't Veld AJ, Schalekamp MA. The effect of balloon angioplasty on hypertension in atherosclerotic renal-artery stenosis. Dutch Renal Artery Stenosis Intervention Cooperative Study Group. N Engl J Med 2000; 342 : 1007-1014.

20 Wheatley K, Ives N, Gray R, Kalra PA, Moss JG, Baigent C, Carr S, Chalmers N, Eadington D, Hamilton G, Lipkin G, Nicholson A, Scoble J. Revascularization versus medical therapy for renal-artery stenosis. N Engl J Med 2009; 361: 1953-1962.

21 Hasebroock KM, Serkova NJ. Toxicity of MRI and CT contrast agents. Expert Opin Drug Metab Toxicol 2009; 5: 403-416.

22 Davis JO, Freeman RH. Mechanisms regulating renin release. Physiol Rev 1976; 56: $1-56$.

23 May AG, De Weese JA, Rob CG. Hemodynamic effects of arterial stenosis. Surgery 1963; 53: 513-524.

24 Radermacher J, Chavan A, Bleck J, Vitzthum A, Stoess B, Gebel MJ, Galanski M, Koch $\mathrm{KM}$, Haller H. Use of Doppler ultrasonography to predict the outcome of therapy for renal-artery stenosis. N Engl J Med 2001; 344: 410-417.

25 Zeller T, Frank U, Muller C, Burgelin K, Sinn L, Bestehorn HP, Cook-Bruns N, Neumann FJ. Predictors of improved renal function after percutaneous stent-supported angioplasty of severe atherosclerotic ostial renal artery stenosis. Circulation 2003; 108: 2244-2249.

26 Garcia-Criado A, Gilabert R, Nicolau C, Real MI, Muntana X, Blasco J, Ganau S, Bru C. Value of Doppler sonography for predicting clinical outcome after renal artery revascularization in atherosclerotic renal artery stenosis. J Ultrasound Med 2005; 24: 1641-1647. 